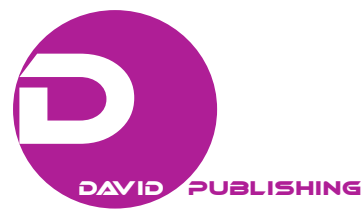

\title{
Is the Productivity-Based Explanation for the Time-Varying US NAIRU Valid?
}

\author{
Hiroshi Yamada \\ Department of Economics \\ Hiroshima University, Japan \\ Gawon Yoon \\ Department of Economics \\ Kookmin University, S. Korea
}

\begin{abstract}
This paper empirically examines whether the productivity-based explanation for the time-varying US NAIRU is valid. We apply a novel filter known as the $1 \mathrm{~d}$ fused lasso. This procedure enables us to estimate the mean trend of the labor productivity as a step function, without specifying the location and number of breaks a priori. This paper reports that although there exists an impressive similarity between the US NAIRU and the US labor productivity, changes in the US labor productivities do not necessarily lead to changes in the US NAIRU.
\end{abstract}

Key words: NAIRU, labor productivity, step function, 1d fused lasso.

\section{Introduction}

What causes the nonaccelerating inflation rate of unemployment (NAIRU) of the US to change over time? A number of theories have been presented to explain this and the explanation based on a mismatch of productivity and wage aspirations, the productivity-based explanation, is one of such theories. ${ }^{1}$ In relation to the explanation, Ball and Mankiw (2002, p. 129) state that, “...a key fact about the productivity acceleration is that it started in the mid-1990s, around the same time that researchers started detecting a decline in the NAIRU. This coincidence suggests a link between the two phenomena." Furthermore, they note that, "If there is a link between shifts in productivity growth and in the NAIRU, it may help explain both the rising NAIRU of the 1970s and the falling NAIRU of the 1990s."

This study aims to contribute to the literature by examining whether the shifts in productivity growth can help explain both the rising NAIRU of the 1970s and the falling NAIRU of the 1990s. If the theory is accurate, changes in the US labor productivities must lead to changes in the US NAIRU. We examine whether such phenomena are observable using a novel procedure. The procedure, known as the $1 \mathrm{~d}$ fused lasso, enables us to estimate the mean trend of the labor productivity as a step function, without specifying the location and number of breaks a priori. We then compare the extracted mean trend with the NAIRU estimated in a companion paper, Yamada and Yoon (2013).

This paper is organized as follows. In Section 2, we briefly review the methodology. In Section 3, we

\footnotetext{
${ }^{1}$ See Ball and Mankiw (2002) and the references therein.

Corresponding author: Hiroshi Yamada, Department of Economics, Hiroshima University.
} 
report our empirical results. In Section 4, we provide concluding remarks.

\section{Methodology}

In this paper, we estimate the mean trend of the US labor productivity growth as a step function. We use a novel filter. The filter estimates $\boldsymbol{x}=\left[x_{1}, x_{2}, \ldots, x_{T}\right]^{\prime}$ from $\boldsymbol{y}=\left[y_{1}, y_{2}, \ldots, y_{T}\right]^{\prime}$ to minimize the following objective function:

$$
\sum_{t=1}^{T}\left(y_{t}-x_{t}\right)^{2}+\lambda \sum_{t=2}^{T}\left|\Delta x_{t}\right|,
$$

where $\Delta x_{t}=x_{t}-x_{t-1}$ and $\lambda>0$ is a tuning parameter. This filter is quite similar to the exponential smoothing (ES) filter, presented in King and Rebelo (1993), of which objective function is

$$
\sum_{t=1}^{T}\left(y_{t}-x_{t}\right)^{2}+\psi \sum_{t=2}^{T}\left(\Delta x_{t}\right)^{2},
$$

where $\psi>0$ is a tuning parameter. However, it has the notable property that it enables us to estimate the mean trend as a step function without specifying the location and number of breaks a priori, whereas the ES filter generally provides a smooth mean trend. With this property, we can estimate the break points of the mean of the labor productivity. Tibshirani and Taylor (2011) refer to this filter as the 1d fused lasso and it is also a special case of the segmented polynomial regression presented in Kim et al. (2009). Note that the relation between the ES filter and the 1d fused lasso corresponds to the relation between the ridge regression of Hoerl and Kennard (1970) and the lasso regression of Tibshirani (1996).

Here, we would like to provide a brief exposition as to why a step function is obtainable. This property is derived from the inclusion of $\ell_{1}$-norm as a penalty in Eq. (1). It generally leads to a sparse solution, and in this setting it makes many entries of $\left[\Delta \hat{x}_{2}, \Delta \hat{x}_{3}, \ldots, \Delta \hat{x}_{T}\right]^{\prime}$ be zero, where $\left[\hat{x}_{1}, \hat{x}_{2}, \ldots, \hat{x}_{T}\right]^{\prime}$ is the extracted mean trend by applying the $1 \mathrm{~d}$ fused lasso. Suppose, for example, $\Delta \hat{x}_{2}=\Delta \hat{x}_{3}=\Delta \hat{x}_{5}=\Delta \hat{x}_{6}=0$ and $\Delta \hat{x}_{4} \neq 0$, then $\hat{x}_{1}=\hat{x}_{2}=\hat{x}_{3}$ and $\hat{x}_{4}=\hat{x}_{5}=\hat{x}_{6}$, but because of $\Delta \hat{x}_{4} \neq 0$, we see $\hat{x}_{3} \neq \hat{x}_{4} .^{2}$

We denote $\boldsymbol{D}$ as the first-order difference matrix such that $\boldsymbol{D} \boldsymbol{x}=\left[x_{2}-x_{1}, x_{3}-x_{2}, \ldots, x_{T}-x_{T-1}\right]^{\prime}$. Explicitly, it is a $(T-1) \times T$ bidiagonal and Toeplitz matrix of which the first row is $[-1,1,0, \ldots, 0]$ :

$$
\boldsymbol{D}=\left[\begin{array}{cccc}
-1 & 1 & & \\
& \ddots & \ddots & \\
& & -1 & 1
\end{array}\right] \text {. }
$$

Then, we define

$$
\lambda_{\max }=2\left\|\left(\boldsymbol{D} \boldsymbol{D}^{\prime}\right)^{-1} \boldsymbol{D} \boldsymbol{y}\right\|_{\infty}
$$

Here, for a vector $\boldsymbol{z}=\left[z_{1}, z_{2}, \ldots, z_{T}\right]^{\prime},\|z\|_{\infty}=\max \left(\left|z_{1}\right|,\left|z_{2}\right|, \ldots,\left|z_{T}\right|\right)$. Then, it is shown that when $\lambda$ is selected as $\lambda \geq \lambda_{\max }$, the extracted mean trend becomes a horizontal line, and the value equals

\footnotetext{
${ }^{2}$ See also Yamada and Jin (2013) and Yamada and Yoon (2014) that uses a similar filter known as the $\ell_{1}$ trend filter.
} 
$\bar{y}=(1 / T) \sum_{t=1}^{T} y_{t}$. This is a notable property because for the ES filter, in contrast, the extracted mean trend that is expressed as

$$
\tilde{\boldsymbol{x}}=\left(\boldsymbol{I}_{T}+\psi \boldsymbol{D}^{\prime} \boldsymbol{D}\right)^{-1} \boldsymbol{y}
$$

becomes a horizontal line when $\psi \rightarrow \infty .^{3}$ Here, $\boldsymbol{I}_{T}$ is the identity matrix of size $T$. Due to this notable property, we select $\lambda$ such that $\lambda=\alpha \lambda_{\text {max }}$, where $0<\alpha<1$. The number of break points generally decreases as the value of $\alpha$ increases.

\section{Empirical Results}

Panel A in Figure 1 depicts the growth rates of the US labor productivity over the period 1960-2011, sourced from the United States Department of Labor (http://data.bls.gov/timeseries/PRS85006092). With the data presented in Panel A in Figure 1, we estimate the mean trend of the US labor productivity to examine whether the shifts in productivity growth can help explain both the rising NAIRU of the 1970s and the falling NAIRU of the 1990s. Panel B in Figure 1 plots the results. Three estimated mean trends corresponding to the cases where $\alpha=0.3,0.4$, and 0.5 are presented. The minimization given in (1) is accomplished by utilizing CVX, which is a Matlab-based modeling system for convex optimization (Grant and Boyd, 2011). Because $\lambda_{\max }$ is about $13.7, \lambda=\alpha \lambda_{\max }$ with $\alpha=0.3,0.4$, and 0.5 are respectively around 4.1 , 5.5 , and 6.9. From this figure, we can observe that, for all values of $\lambda$, the mean trend of US labor productivity declined in 1974 (at Point a) and rose in 1996 (at Point b) and 1998 (at Point c), which may correspond to the rising NAIRU of the 1970s and the falling NAIRU of the 1990s. ${ }^{4}$ We also conducted an additional empirical examination with another labor productivity data, taken from the John Fernald's website (http://www.frbsf.org/economics/economists/staff.php?jfernald). The data denoted by dLP is used. As a result, the qualitatively almost the same empirical evidence are observed. ${ }^{5}$

Panel A in Figure 2 provides the time-varying NAIRU estimate taken from Yamada and Yoon (2013). The method used to obtain this is similar to the minimization given in (1). The NAIRU estimate presented in this panel corresponds to the case where $\alpha=0.4$, although for this case $\lambda_{\max }$ is different from (3). See Yamada and Yoon (2013) for more details. From this figure, we can observe that both the rising NAIRU of the 1970s and the falling NAIRU of the 1990s are detected at Point A (1973), Point B (1994), and Point C (1997). ${ }^{6}$ Panel $\mathrm{B}$ in Figure 2 shows the time-varying mean of the US labor productivity where $\alpha=0.4$. Note that, in this figure, for comparison, the sign is reversed. From these panels, it is not observable that the changes in labor productivity led to the changes in the NAIRU. Conversely, the changes in labor productivity lagged behind the changes in the NAIRU by one or two years. For example, Point $b$ lags behind Point B by two years. This observation can be seen as an empirical evidence against the productivity-based explanation of the time-varying

\footnotetext{
${ }^{3}$ This can be derived as follows: $\lim _{\psi \rightarrow \infty} \tilde{\boldsymbol{x}}=\boldsymbol{y}-\lim _{\psi \rightarrow \infty} \boldsymbol{D}^{\prime}\left(\psi^{-1} \boldsymbol{I}_{T-1}+\boldsymbol{D} \boldsymbol{D}^{\prime}\right)^{-1} \boldsymbol{D} \boldsymbol{y}=\left\{\boldsymbol{I}_{T}-\boldsymbol{D}^{\prime}\left(\boldsymbol{D} \boldsymbol{D}^{\prime}\right)^{-1} \boldsymbol{D}\right\} \boldsymbol{y}=\boldsymbol{\imath}\left(\boldsymbol{\imath}^{\prime} \boldsymbol{\imath}\right)^{-1} \boldsymbol{\imath}^{\prime} \boldsymbol{y}=\bar{y} \boldsymbol{\imath}$, where $\boldsymbol{\imath}$ is the $T \times 1$ vector of ones.

${ }^{4}$ We tried $\alpha=0.2$ and 0.6 as well. We observed that, for both cases, Points a and $\mathrm{b}$ are detected, but Point $\mathrm{c}$ is not detected when $\alpha=0.6$.

${ }^{5}$ They are available from the authors upon request.

${ }^{6}$ Although only one estimate corresponding to the case $\alpha=0.4$ is presented in the panel, we examined the cases $\alpha=0.3,0.4$, 0.5 , and 0.6. As a result of our examination, we observed that, for all cases, Point A was detected. In addition, Point B was detected except in the case where $\alpha=0.6$. Point $\mathrm{C}$, however, is not detected for the cases $\alpha=0.5$ and 0.6 .
} 
US NAIRU. Still, we would like to note that, as shown in this figure, there exists an impressive similarity between the US NAIRU and the US labor productivity.

\section{Concluding Remarks}

In this paper, we examined whether shifts in productivity growth can help explain both the rising NAIRU of the 1970s and the falling NAIRU of the 1990s, using a novel filter known as the 1d fused lasso. As a result of our empirical investigation, we found that although there exists an impressive similarity between the US NAIRU and the US labor productivity, changes in the US labor productivities do not necessarily lead to changes in the US NAIRU, which may be regarded as an empirical evidence against the productivity-based explanation. Of course, we understand that our empirical evidence is not sufficient to establish a definite conclusion and that, to reach such a conclusion, more empirical results are required.

Pane1 A: The US labor productivity

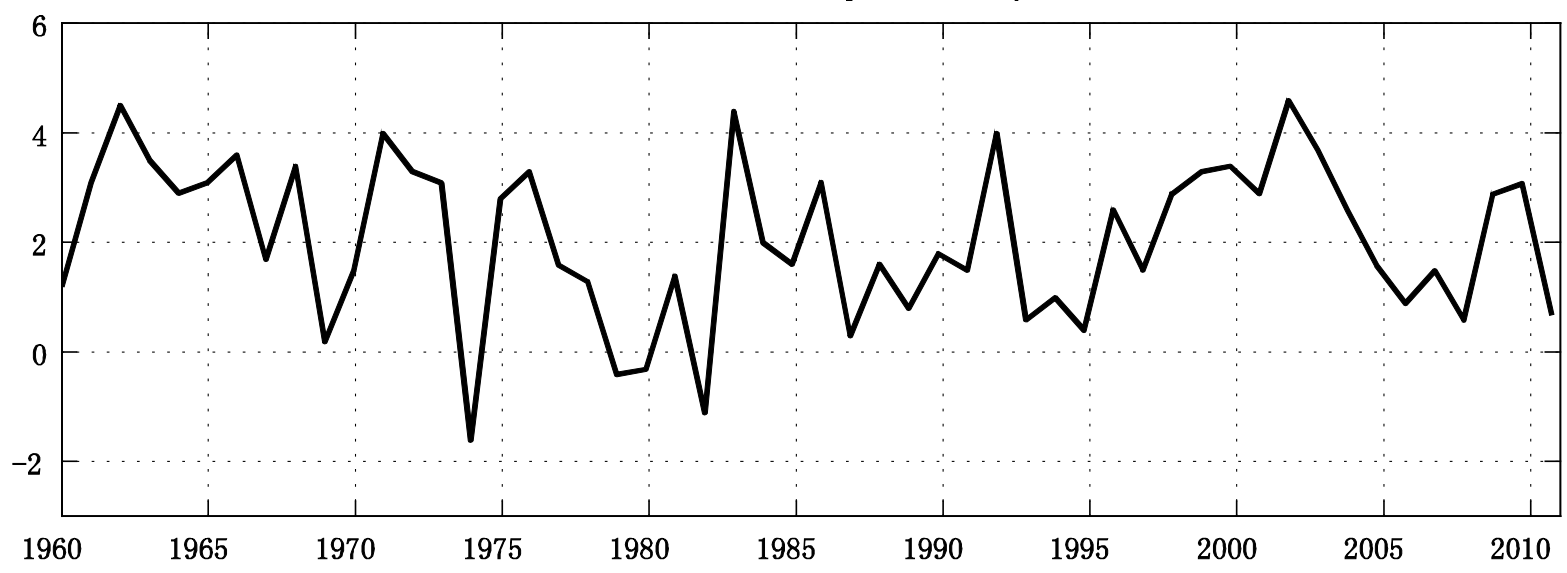

Panel B: The time-varying mean of the US labor productivity

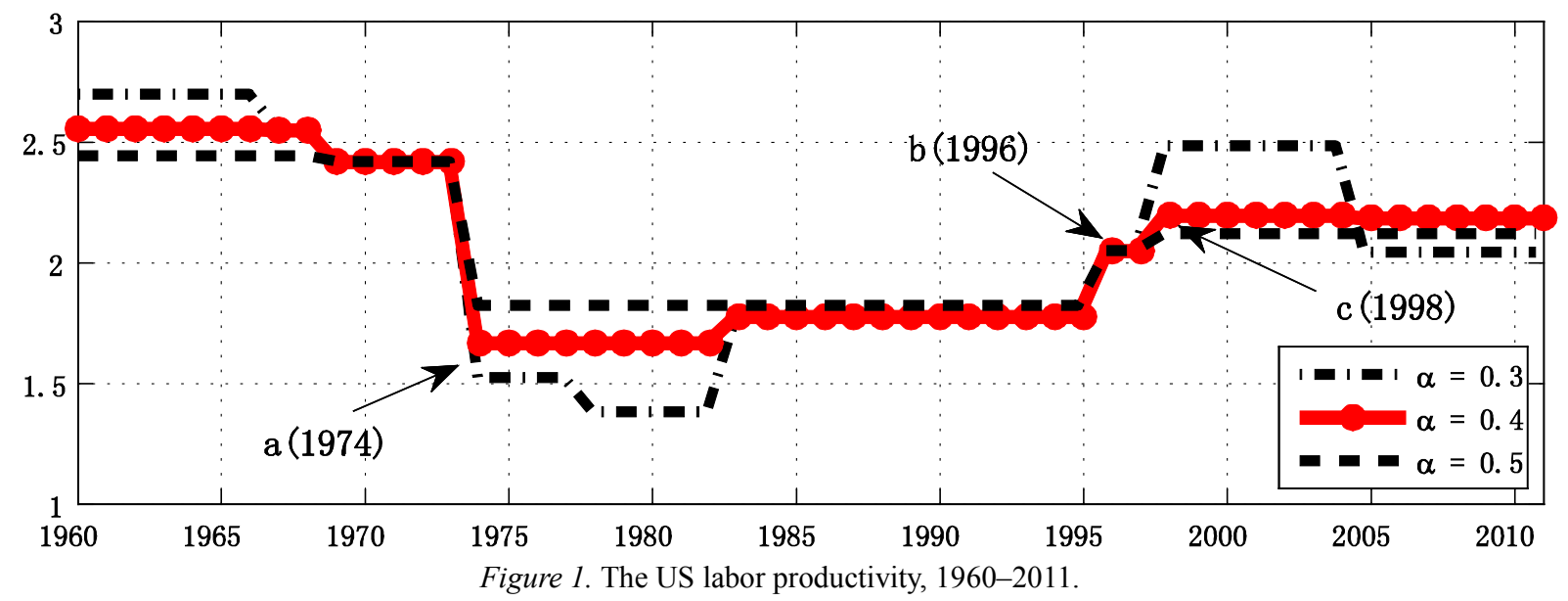




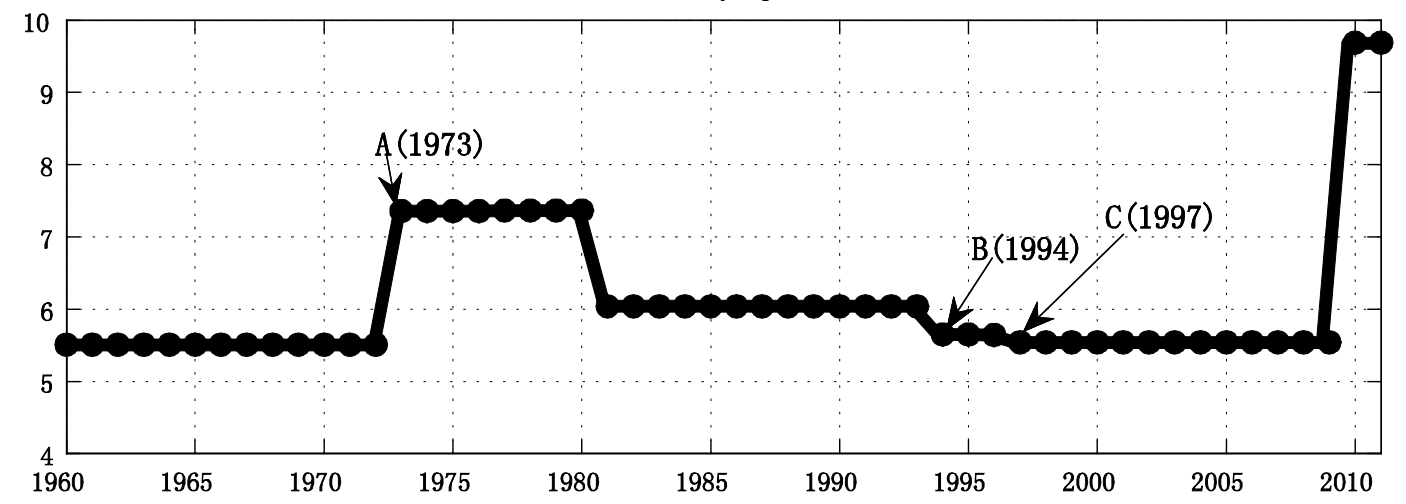

Panel B: The time-varying mean of the US labor productivity (reversed sign)

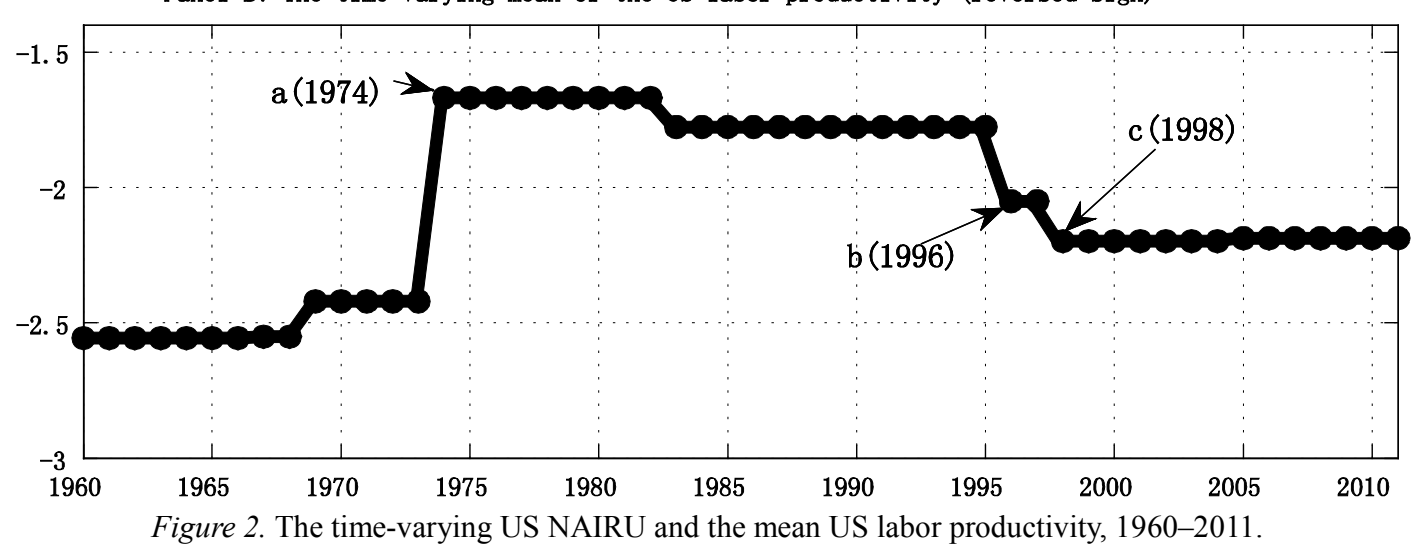

\section{Acknowledgement}

We thank John Fernald for providing his data set. Hiroshi Yamada's work was partly supported by a grant-in-aid from the Zengin Foundation for Studies on Economics and Finance and by KAKENHI (22530272). Gawon Yoon's work was supported by the Research Program of Kookmin University in Korea.

\section{References}

Ball, L. and G. Mankiw, 2002, The NAIRU in theory and practice, Journal of Economic Perspectives, 16, 4, 115-136.

Grant, M. and S. Boyd, 2011, CVX: Matlab software for disciplined convex programming, Version 1.21, available at: (http://cvxr.com/cvx).

Hoerl, A.E. and R.W. Kennard, 1970, Ridge regression: Biased estimation for nonorthogonal problems, Technometrics 12, 1, $55-67$.

Kim, S., K. Koh, S. Boyd, and D. Gorinevsky, 2009, $\ell_{1}$ trend filtering, SIAM Review, 51, 2, 339-360.

King, R.G. and S.T. Rebelo, 1993, Low frequency filtering and real business cycles, Journal of Economic Dynamics and Control, $17,1-2,207-231$.

Tibshirani, R., 1996, Regression shrinkage and selection via lasso, Journal of Royal Statistical Society B, 58, 1, $267-288$.

Tibshirani, R.J. and J. Taylor, 2011, The solution path of the generalized lasso, Annals of Statistics, 39, 3, $1335-1371$.

Yamada, H. and L. Jin, 2013, Japan's output gap and $\ell_{1}$ trend filtering, Empirical Economics, 45, 1, 81-88.

Yamada, H. and G. Yoon, 2013, Measuring the US NAIRU as a step function: A new approach, submitted for publication.

Yamada, H. and G. Yoon, 2014, When Grilli and Yang meet Prebisch and Singer: Piecewise linear trends in primary commodity prices, Journal of International Money and Finance, 42, 193-207. 